\title{
Effect of an expansion chamber on the propagation of sound in circular ducts
}

\author{
A. I. El-Sharkawy and Ali H. Nayfeh \\ Engineering Science and Mechanics, Virginia Polytechnic Institute and State University, Blacksburg, \\ Virginia 24061 \\ (Received 24 January 1977)
}

\begin{abstract}
An analytical and experimental study is presented of sound propagation through a circular duct in the presence of an expansion chamber. Results are presented for various sound frequencies, expansion ratios, and chamber lengths. It is found that the analytical results are in good agreement with the experimental results.
\end{abstract}

PACS numbers: $43.20 . \mathrm{Mv}$

\section{INTRODUCTION}

The reflection of sound waves due to a change in the cross section of a circular duct was first described by Miles ${ }^{1}$; he determined the reflection coefficient due to a discontinuity by calculating the pressure distribution in the vicinity of the discontinity. Davis et al. ${ }^{2}$ matched the pressure and volume velocity across the discontinuities to derive an equation for the transmission loss of an "expansion-chamber silencer." Their analysis considered only plane causes. Therefore, their analysis is limited to low frequencies where all modes except the lowest mode are cutoff.

In terms of the sound wavelength $\lambda$, their equation is valid only for $\lambda \leq 0.82 b$, where $b$ is the radius of the expansion chamber. Their experimental results showed a decrease in the attenuation at high frequencies, indicating the presence of undamped modes, and therefore reducing seriously the silencer effectiveness.

Lansing and Zorumski ${ }^{3}$ investigated the effect of a continuous variation of the wall admittance of a uniform duct by approximating the duct by a series of subsections. Alfredson ${ }^{4}$ investigated the effect of a continuous variation of the duct cross section by dividing the duct into a series of uniform subsections. His analysis was limited to nonspinning modes. Young and Crocker ${ }^{5}$ used the finite-element method to predict the transmission loss of expansion-chamber mufflers. Their results showed that the stability of the solution depends on the number of elements considered. Craggs ${ }^{6}$ applied the same method to calculate the transmission loss of expansion-chamber mufflers and compared his results with a plane-wave analysis. His results are compared in this paper with the present analysis. For a more detailed review of waves propagating in nonuniform ducts, see Nayfeh et $a l .{ }^{7}$ and Nayfeh. ${ }^{8}$

This paper aims at investigating the effect of an expansion-chamber silencer on the sound propagation and reflection in circular ducts. Numerical results are presented by including as many modes as needed for convergence. The effects of expansion ratio, chamber length, and sound frequency are illustrated. Experiments are conducted to validate the theoretical results. It is found that the analytical results are in good agreement with the experimental results.

\section{THEORETICAL ANALYSIS}

We consider linear sound waves propagating in a circular hard-walled duct containing an expansion chamber as shown in Fig. 1. The tailpipe is assumed to be anechoically terminated, and viscous effects are neglected. With these assumptions, one can express the sound pressure at any point $(x, r, \theta)$ as

$$
p_{r}=\sum_{m=0}^{\infty} \sum_{n=0}^{\infty} A_{m n} J_{m}\left(\lambda_{m n} r\right) \exp \left[i\left(k_{m n} x-\omega t+m \theta\right)\right]
$$

for waves traveling to the right and

$$
p_{l}=\sum_{m=0}^{\infty} \sum_{n=0}^{\infty} B_{m n} J_{m}\left(\lambda_{m n} r\right) \exp \left[i\left(-k_{m n} x-\omega t+m \theta\right)\right]
$$

for waves traveling to the left. Hence, the axial particle velocity is

$$
u_{r}=\sum_{m=0}^{\infty} \sum_{n=0}^{\infty} \frac{k_{m n}}{\rho \omega} A_{m n} J_{m}\left(\lambda_{m n} r\right) \exp \left[i\left(k_{m n} x-\omega t+m \theta\right)\right]
$$

for waves traveling to the right and

$$
u_{l}=-\sum_{m=0}^{\infty} \sum_{n=0}^{\infty} \frac{k_{m n}}{\rho \omega} B_{m n} J_{m}\left(\lambda_{m n} r\right) \exp \left[i\left(-k_{m n} x-\omega t+m \theta\right)\right],
$$

where the $x$ direction is taken as the direction of propagation of the incident wave. If the radius of the pipe is $a$ and that of the expansion chamber is $b$, then

$$
\begin{aligned}
& \lambda_{1 m n}=a^{-1} \alpha_{m n}, \quad \lambda_{2 m n}=b^{-1} \alpha_{m n} \\
& k_{1 m n}^{2}=(\omega / c)^{2}-\lambda_{1 m n}^{2}, \quad k_{2 m n}^{2}=(\omega / c)^{2}-\lambda_{2 m n}^{2},
\end{aligned}
$$

where $\omega$ is the circular frequency of the sound, $c$ is the speed of sound, and the $\alpha_{m n}$ are the roots of $J_{m}^{\prime}(\alpha)=0$.

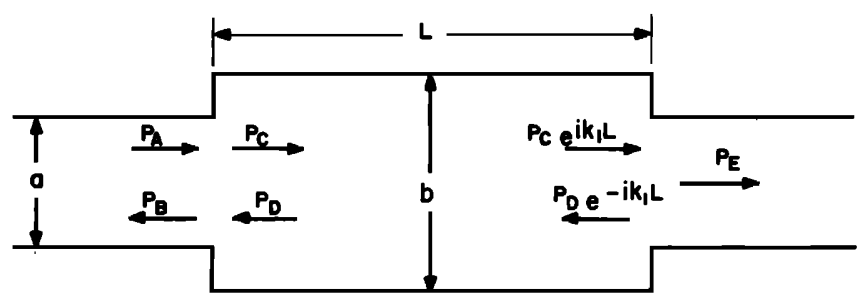

FIG. 1. Expansion chamber model. 
Following Miles, we match the waves in the different sections by imposing the following conditions: (a) equality of pressure across each discontinuity for $0 \leq r \leq a$, (b) equality of volume velocity across each dis continuity for $0 \leq r \leq a$, and (c) vanishing of volume ve- locity on both ends of the chamber for $a \leq r \leq b$. Applying the above conditions, using the orthogonality relations of the Bessel functions and separating the variations in $\theta$, we obtain the following equations:

$$
\begin{aligned}
& \frac{1}{2}\left(A_{m s}+B_{m s}\right)\left[\frac{\alpha_{m s}^{2}-m^{2}}{\lambda_{1 m s}^{2}} J_{m}^{2}\left(\alpha_{m s}\right)\right]=\sum_{n=0}^{\infty}\left(C_{m n}+D_{m n}\right) \frac{a \lambda_{2 m n} J_{m}\left(\alpha_{m s}\right) J_{m}^{\prime}\left(\lambda_{2 m n} a\right)}{\lambda_{1 m s}^{2}-\lambda_{2 m n}^{2}} \\
& \sum_{n=0}^{\infty}\left(A_{m n}-B_{m n}\right) k_{1 m n} \frac{a \lambda_{2 m s} J_{m}\left(\alpha_{m n}\right) J_{m}^{\prime}\left(\lambda_{2 m} a\right)}{\lambda_{1 m n}^{2}-\lambda_{2 m n}^{2}}=\frac{1}{2}\left(C_{m s}-D_{m s}\right) k_{2 m s} \frac{\alpha_{m s}^{2}-m^{2}}{\lambda_{2 m s}^{2}} J_{m}^{2}\left(\alpha_{m s}\right) \\
& \sum_{n=0}^{\infty}\left[C_{m n} \exp \left(i k_{2 m n} L\right)+D_{m n} \exp \left(-i k_{2 m n} L\right)\right] \frac{a \lambda_{2 m n} J_{m}\left(\alpha_{m s}\right) J_{m}^{\prime}\left(\lambda_{2 m n} a\right)}{\lambda_{1 m s}^{2}-\lambda_{2 m n}^{2}}=\frac{1}{2} E_{m s} \exp \left(i k_{1 m s} L\right)\left[\frac{\alpha_{m s}^{2}-m^{2}}{\lambda_{1 m s}^{2}} J_{m}^{2}\left(\alpha_{m s}\right)\right] \\
& \frac{1}{2}\left[C_{m s} \exp \left(i k_{2 m s} L\right)-D_{m s} \exp \left(-i k_{2 m s} L\right)\right] k_{2 m s} \frac{\alpha_{m s}^{2}-m^{2}}{\lambda_{1 m s}^{2}} J_{m}^{2}\left(\alpha_{m s}\right)=\sum_{n=0}^{\infty} E_{m n} k_{1 m n} \exp \left(i k_{1 m n} L\right) \frac{a \lambda_{2 m s} J_{m}\left(\alpha_{m n}\right) J^{\prime}\left(\lambda_{2 m n} a\right)}{\lambda_{1 m n}^{2}-\lambda_{2 m s}^{2}}
\end{aligned}
$$

Equations (6)-(9) represent four sets of equations containing an infinite number of unknowns for each spinning mode (i.e., $m$ ) and each input. To solve these equations we need to truncate the series after a finite number of terms. The input is taken to be corresponding to the experimental value. The other calculated pressures are referred to the incident wave. The numerical results were performed by including as many terms in the truncated series as needed for convergence. It was found that, at most, five terms were sufficient to yield a $0.1 \%$ accuracy for all the range of parameters presented in this paper.

After solving Eqs. (6)-(9) for the amplitudes, we calculated the pressures on both sides of the discontinuity from Eqs. (1) and (2). The results presented in this paper cover a wide range of sound frequencies, expansion ratios, and chamber lengths.

\section{EXPERIMENT}

The test apparatus used in this investigation is shown schematically in Fig. 2. The sound was generated by an audio signal generator feeding into a $60-\mathrm{W}$ amplifier and conducted to the system by means of a coaxial loud speaker connected to the pipe by a conical coupling.
The sound which passed through the expansion chamber continued down through the tailpipe (of the same diameter as the leading pipe) to the termination, which consisted of several feet of loosely packed cotton surrounded by a box filled with sound absorbing material. The section of the tube between the loudspeaker and the chamber, called the high-pressure side, was surrounded by a sound-insulating material to minimize the radiation and conduction of sound to and from the surroundings. This was necessary to minimize the system noise. The propagating signal was processed by means of a frequency analyzer, a valve voltmeter, and a level recorder. The measuring microphone was traversed axially and radially along the test section. A reference probe was located at the entrance of the chamber and connected to the driving system by a compression loop in order to maintain a constant pressure level at this point.

The test matrix is shown in Table I. It was chosen to determine the influence of expansion ratio and chamber length on the propagation and reflection of sound. Each run was performed for a number of frequencies.

\section{RESULTS AND DISCUSSIONS}

Figure 3 shows a comparison among the present method, the finite-element method, ${ }^{6}$ and plane-wave theory. ${ }^{2}$

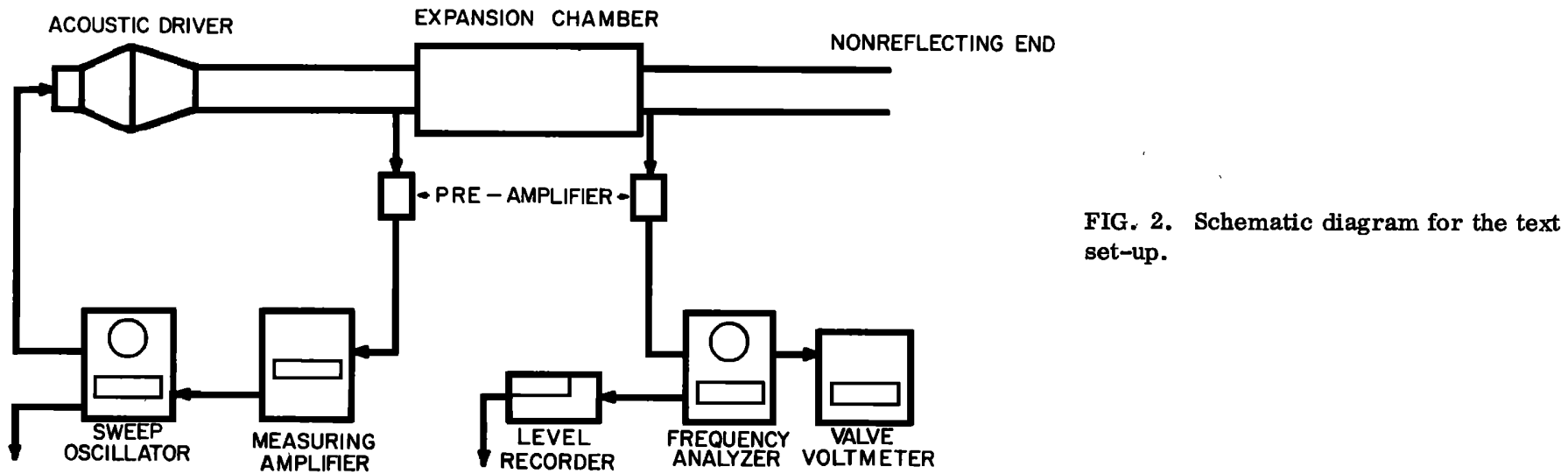


TABLE I. Test matrix.

\begin{tabular}{|c|c|c|c|}
\hline $\begin{array}{l}\text { Run } \\
\text { No. }\end{array}$ & $\begin{array}{l}a \\
\text { (in.) }\end{array}$ & $b / a$ & $L / a$ \\
\hline 1 & 5 & 2 & 2.4 \\
\hline 2 & 5 & 3 & 2.4 \\
\hline 3 & 5 & 4 & 2.4 \\
\hline 4 & 5 & 2 & 3.0 \\
\hline 5 & 5 & 3 & 3.0 \\
\hline 6 & 5 & 4 & 3.0 \\
\hline 7 & 5 & 2 & 3.6 \\
\hline 8 & 5 & 3 & 3.6 \\
\hline 9 & 5 & 4 & 3.6 \\
\hline 10 & 4 & 3 & 3.0 \\
\hline 11 & 5 & 3 & 3.0 \\
\hline 12 & 6 & 3 & 3.0 \\
\hline
\end{tabular}

For comparison purposes, the transmission loss was calculated as $-20 \log _{10} P_{E}$, and in the case of plane-wave theory, it is calculated from the equation ${ }^{2}$

$$
\mathrm{TL}=10 \log _{10}\left[1+\frac{1}{4}\left(\frac{b^{2}}{a^{2}}-\frac{a^{2}}{b^{2}}\right) \sin ^{2} \frac{\omega L}{c}\right] .
$$

At low frequencies and small chamber diameter-tolength ratios $(b / L)$, a general agreement is observed between the three approaches. In the present analysis, sharp peaks start to form for $(b / L)>0.8$. These peaks occur at frequencies slightly below those at which the first radial mode cuts on. Then, the transmission loss falls rapidly owing to the presence of the new mode. In the finite-element method, the deviation from plane-wave theory starts at frequencies lower than those predicted from the present analysis. As more modes are cut on, more finite elements are needed to resolve the propagating waves, and hence produce the desired accuracy. Thus, it is expected that increasing the number of finite elements will lead to better agreement between the finiteelement results and the present analysis at high frequencies.

Figure 3 shows that the transmission loss predicted
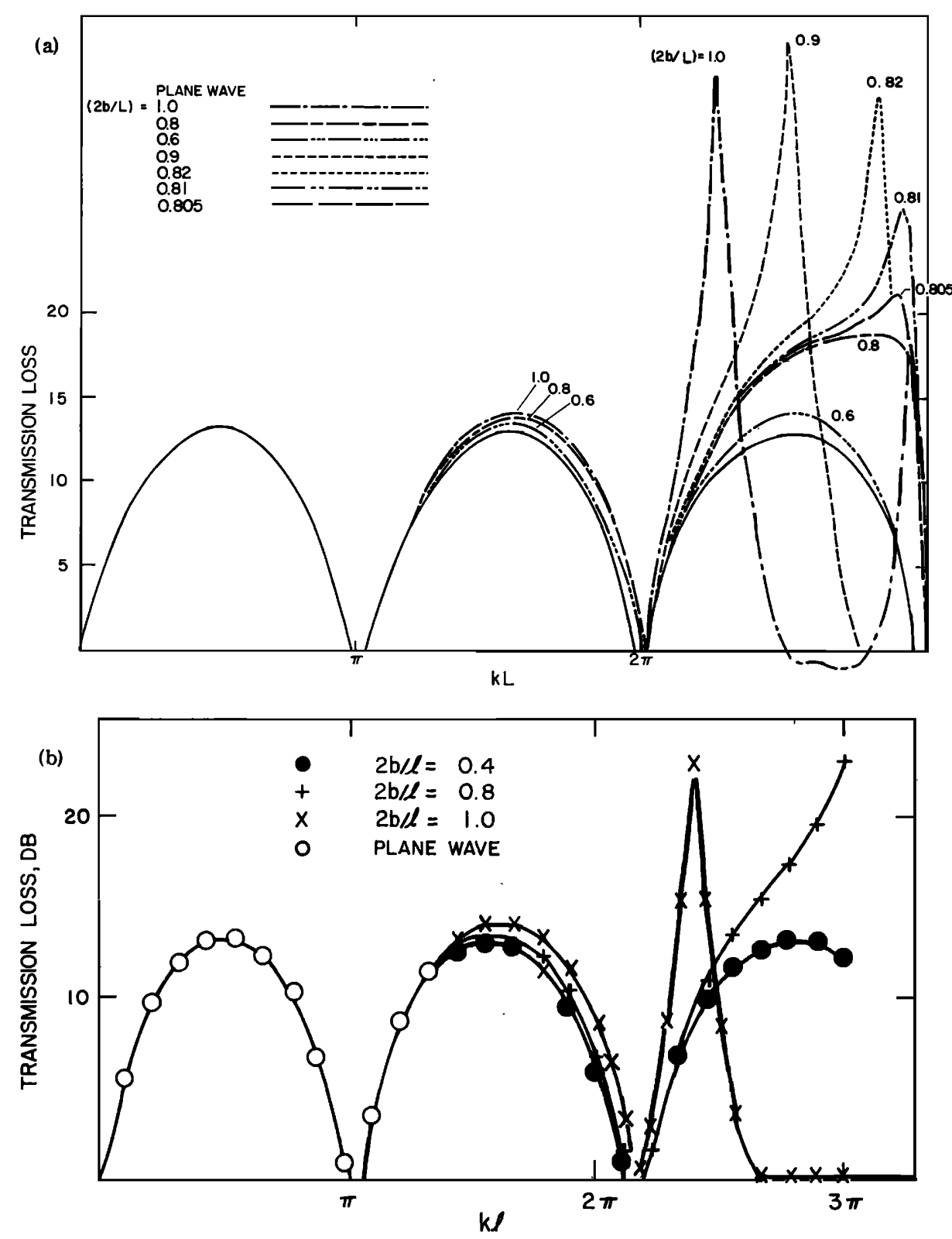

FIG. 3. Comparison of the present analysis with plane-wave theory and the finiteelement method-(a): present analysisand plane-wave theory, (b) the finite-element method and the plane-wave theory. ${ }^{6}$ 


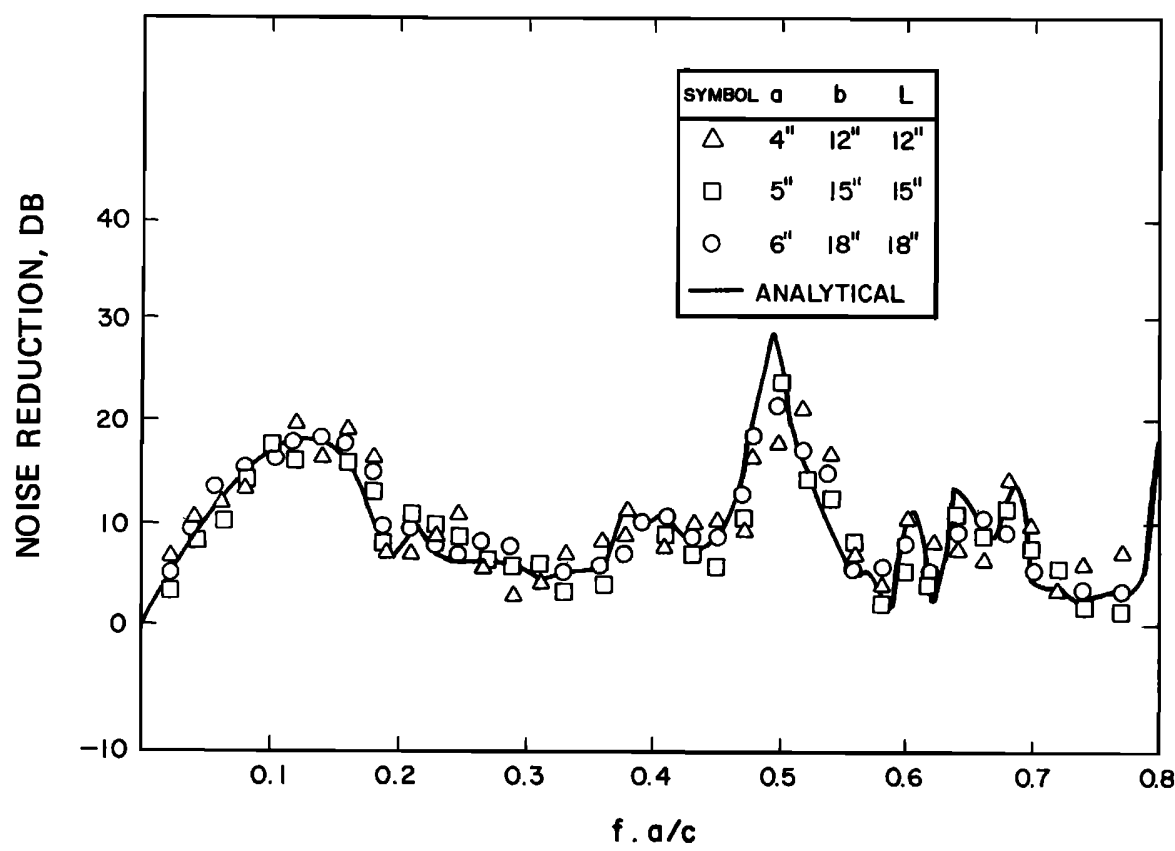

FIG. 4. Reproducibility of experimental results and comparison with present analysis.

by plane-wave theory, Eq. (10), increases with increasing chamber diameter. For a given length, the maximum transmission loss occurs at the frequencies $\frac{1}{2}\left(n+\frac{1}{2}\right) \mathrm{CL}^{-1}$, where $n$ is an integer. Furthermore, the maximum transmission loss is independent of chamber length. Figure 3 shows that including higher-order modes besides the plane mode leads to a nonperiodic variation of the transmission loss with frequency, especially at high frequencies.

To compare the present analysis with our experimental results, we calculated the noise reduction $20 \log _{10}\left(P_{A}+P_{B}\right) / P_{E}$ for a number of frequencies spanning the interval [20-2000 Hz] covered in the experiments and for all configurations shown in Table I.
The results are presented as a function of the dimensionless frequency $(f a / c)$, where $a$ is the pipe radius and $c$ is the speed of sound. We note that at the high end of the frequency interval considered and for the highest expansion ratio, two radial modes besides the plane mode propagate unattenuated in the expansion chamber.

In all the experimental runs, the incident wave is a plane wave. Since the pipes have circular cross sections and since they are symmetric with respect to the silencer axis, no asymmetric modes are excited.

Figures 4-13 compare the experimental and theoretical results and show the influence of the silencer configuration and sound frequency on the noise reduction.

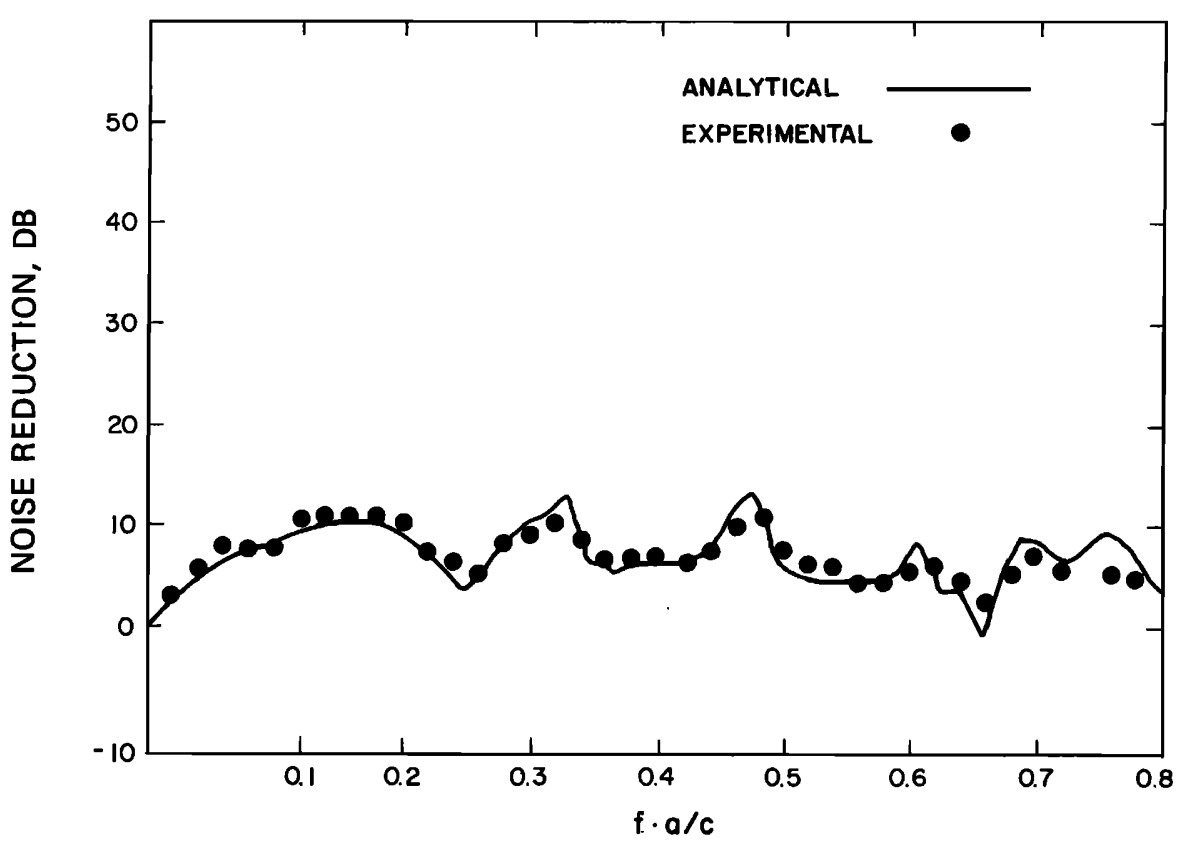

FIG. 5. Comparison of present analytical and experimental results for $(b / a)=2$ and $(L / a)=2.4$. 


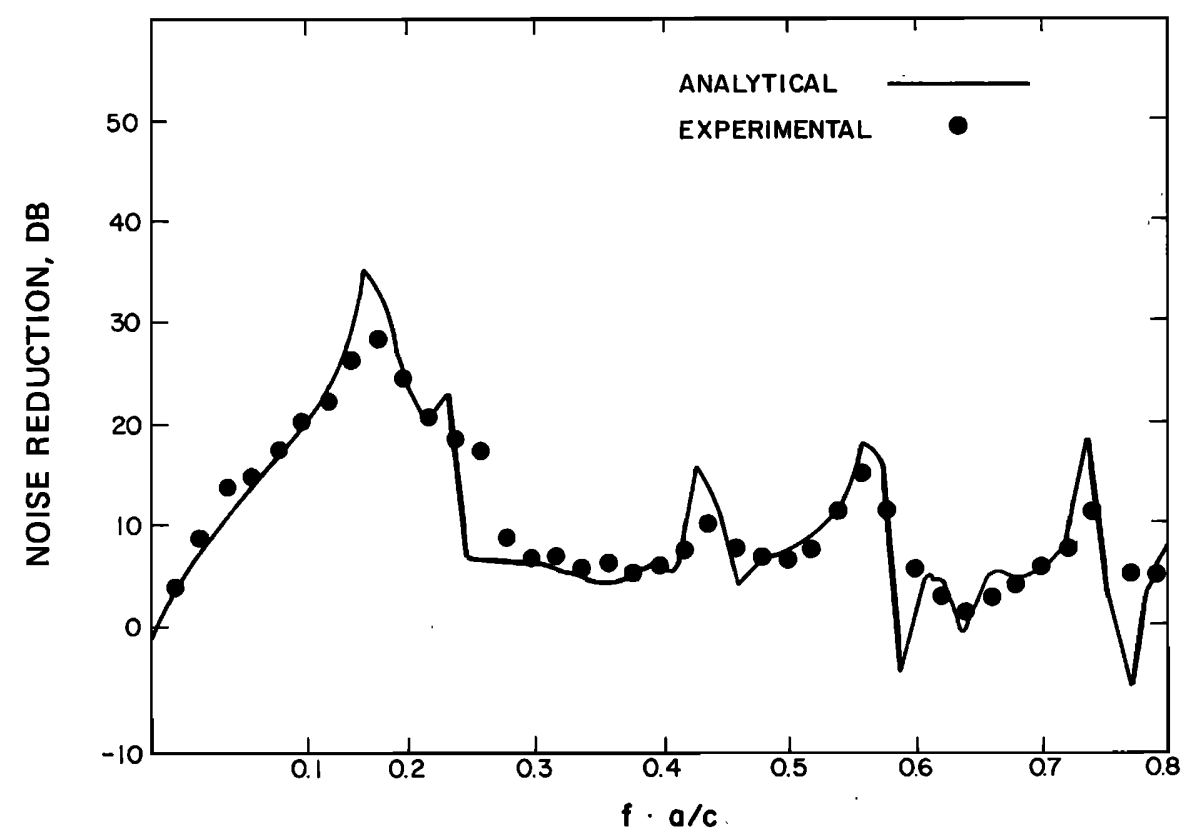

FIG. 6. Comparison of present analytical and experimental results for $(b / a)=3$ and $(L / a)=2.4$.
In the present inviscid hard-walled theory, the noisereduction loss is a function of the three dimensionless parameters $(b / a),(L / a)$, and $(f a / c)$. Figure 4 shows the reproducibility of the experimental results if one keeps these dimensionless parameters fixed while varying the dimensions of the silencer. The experimental results show that, at high frequencies, the noise reduction depends on the dimensions of the silencer although the dimensionless parameters are fixed. This may be due to the neglected viscous effects. Figures 4-13 show good agreement between the theoretical and experimental results for all frequencies and configurations considered.

In contrast with plane wave theory, Figs. 5, 8, 11; Figs. 6, 9, 12; and Figs. 7, 10, and 13 show that the maximum noise reduction is a function of the chamber length. Moreover, for a given chamber length the max- ima of the noise reduction occur at frequencies different from those predicted by plane-wave theory.

The effect of varying the expansion ratio $b / a$ can be seen by comparing the figures in each of the three groups: Figures 5-7, Figs. 8-10, and Figs. 11-13. In each group, the expansion ratio was varied to include 2-4. These ratios were chosen because they are representative of the values suitable for application in automotive silencers. Figures 5 and 6 show that increasing the expansion ratio from 2 to 3 results in an increase in the maximum noise reduction. However, as the expansion ratio increases to 4 , the maximum transmission loss decreases. As the expansion ratio increases, Figs. 5-13 show that the noise-reduction bands become, in general, narrower as the frequency increases. This can be explained by the following argument. Increasing

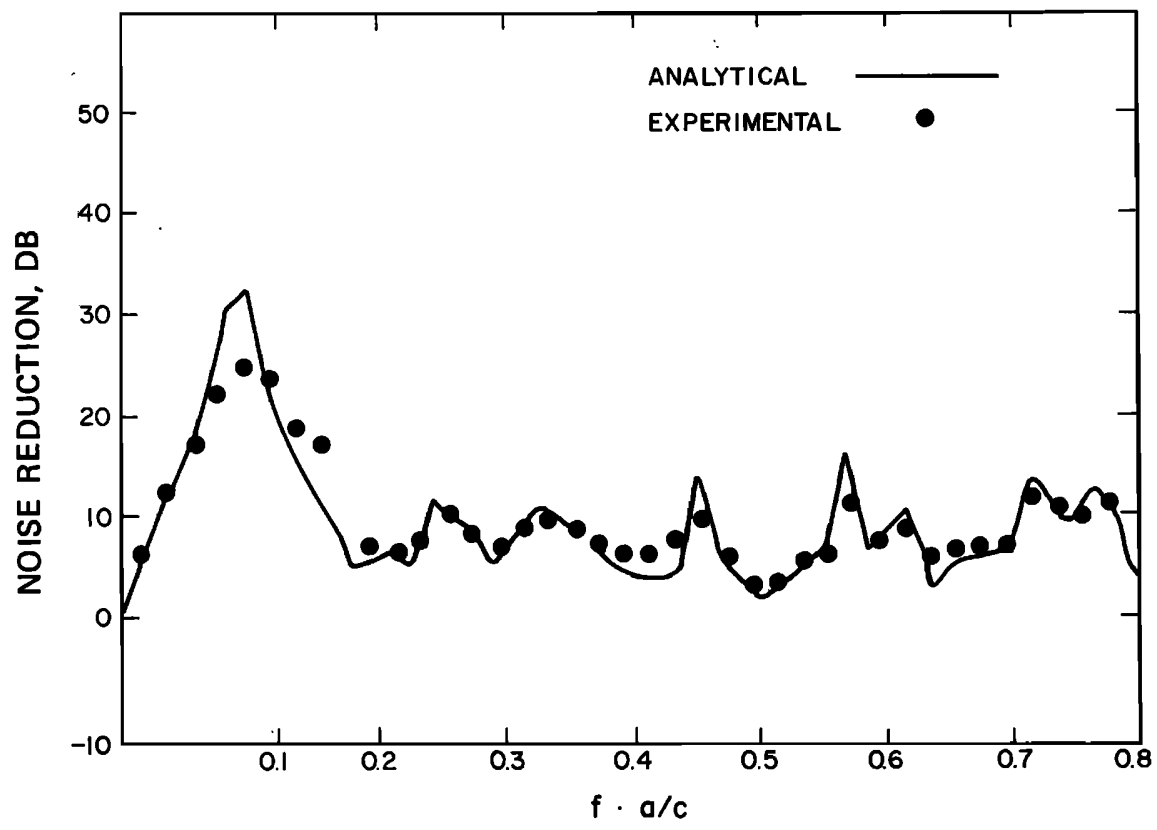

FIG. 7. Comparison of present analytical and experimental results for $(b / a)=4$ and $(L / a)=2.4$. 


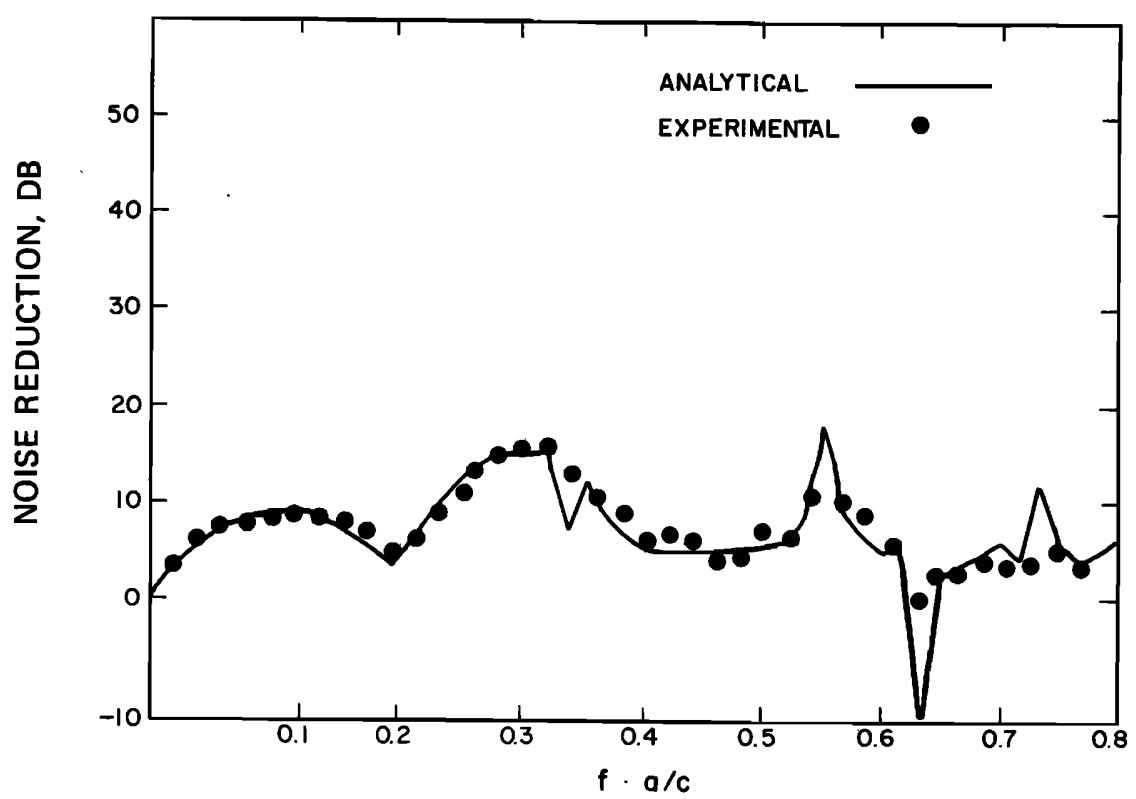

FIG. 8. Corr.parison of present analytical and experimental results for $(b / a)=2$ and $(L / a)=3.0$.

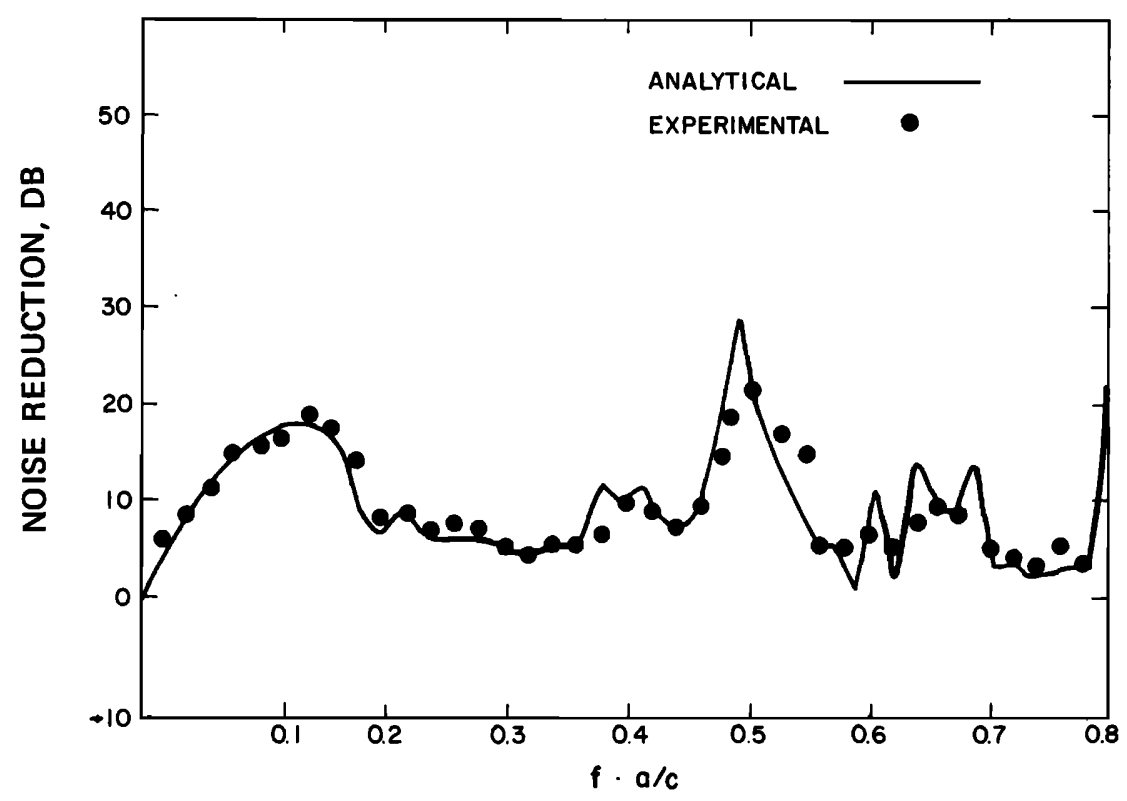

FIG. 9. Comparison of present analytical and experimental results for $(b / a)=3$ and $(L / a)=3.0$.

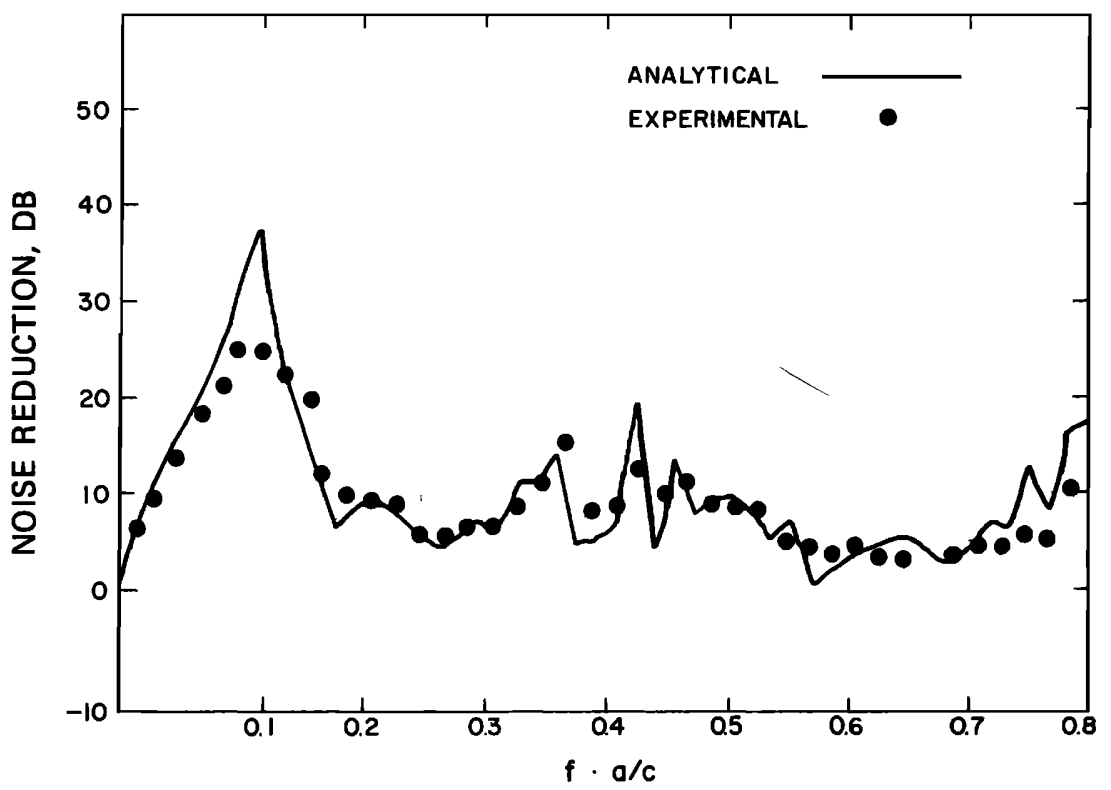

FIG. 10. Comparison of present analytical and experimental results for $(b / a)=4$ and $(L / a)=3.0$. 

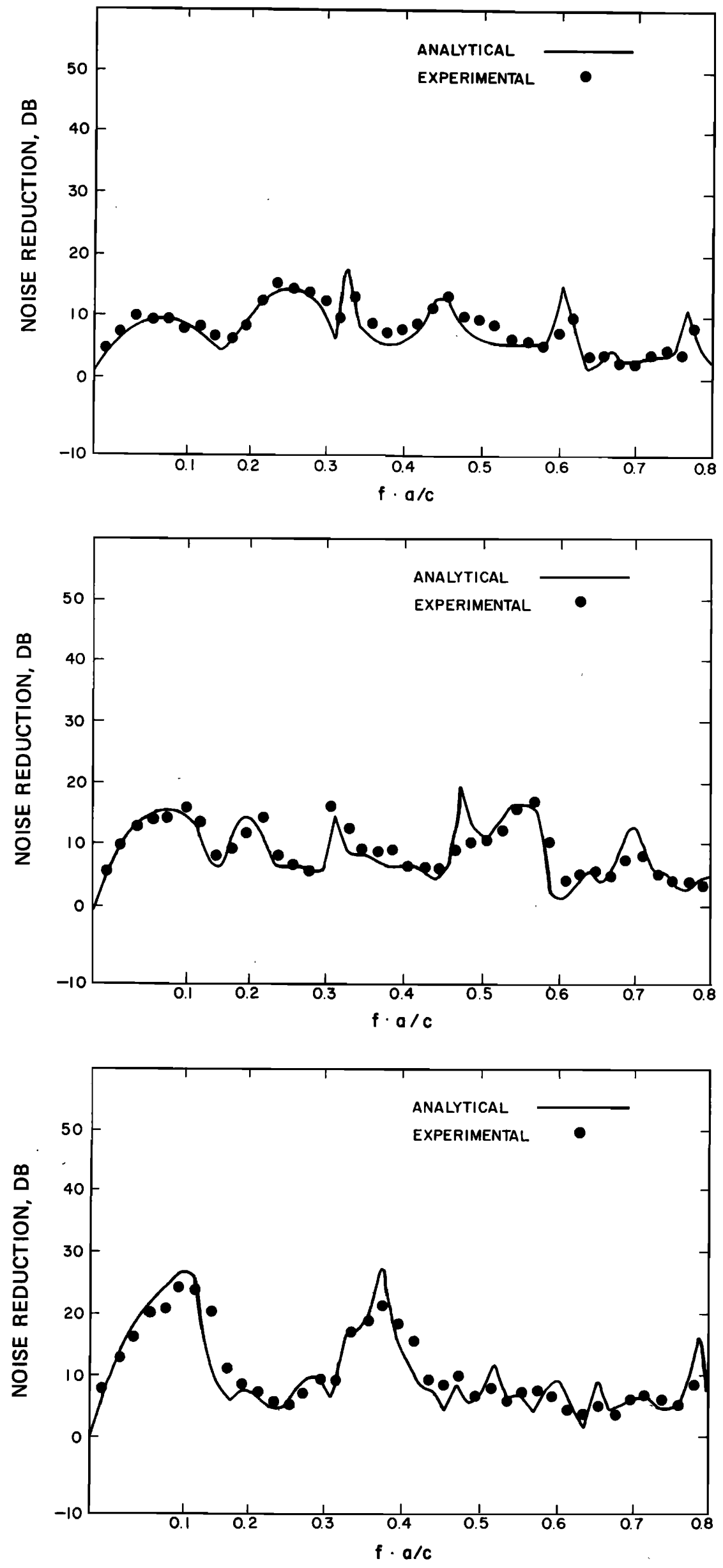

FIG. 11. Comparison of present analytical and experimental results for $(b / a)=2$ and $(L / a)=3.6$.

FIG. 12. Comparison of present analytical and experimental results for $(b / a)=3$ and $(L / a)=3.6$.

FIG. 13. Comparison of present analytical and experimental results for $(b / a)=4$ and $(L / a)=3.6$. 
the expansion ratio causes a corresponding increase in the reflection of the sound at the first and second discontinuities resulting in an increase in the noise reduction. However, when the expansion ratio increases to a value at which a mode is cut on in the expansion chamber, the noise reduction experiences a drop. A further increase in the expansion ratio causes an increase in the noise reduction until another mode is cut on, etc.

The effect of varying the length of the expansion chamber on the noise reduction spectrum can be seen by comparing the curves in each of the following three groups of figures: Figures 5, 8, and 11; Figs. 6, 9, and 12; and Figs. 7, 10, and 13. In each of these groups, the expansion ratio is fixed while the chamber length is varied to include $(L / a)=2.4,3.0$, and 3.6. It can be seen that increasing the chamber length results in a narrowing of the first noise-reduction band, an increase in the maximum noise reduction, and a decrease in the noise reduction at high frequencies.

\section{CONCLUDING REMARKS}

The present analysis reveals that the performance of an expansion chamber is greatly affected by the expan- sion ratio, the sound frequency and to a lesser extent the chamber length. This conclusion was verified experimentally. It is shown that plane-wave theory is valid only at low sound frequencies and small expansion ratios. It is also concluded that expansion-chamber mufflers are efficient in attenuating low-frequency sound, which make them ideal for automotive silencing applications.

${ }^{1} J$. Miles, J. Acoust. Soc. Am. 16, 14-19 (1944).

${ }^{2}$ D. D. Davis, Jr., G. M. Stokes, D. Moore, and G. L. Stevens, Jr., NACA Rep. No. 1192 (1954).

${ }^{3}$ D. L. Lansing and W. E. Zorumski, J. Sound Vib. 27, 85-100 (1973).

${ }^{4}$ R. J. Alfredson, J. Sound Vib. 23, 433-442 (1972).

${ }^{5}$ C. I. J. Young and M. J. Crocker, J. Acoust. Soc. Am. 57, 144-148 (1975).

${ }^{6}$ A. Craggs, J. Sound Vib. 48, 377-392 (1976).

${ }^{7}$ A. H. Nayfeh, AIAA J. 13, 130-153 (1975).

${ }^{8}$ A. H. Nayfeh, "Advances in Engineering Science," in Proceedings of the 13th Anmual Meeting (Society of Engineering Science, Hampton, VA, 1976), Vol. 3, pp. 821-834 (NASA. CP-2001). 